\title{
The TIR Domain Containing Locus of Enterococcus faecalis Is Predominant among Urinary Tract Infection Isolates and Downregulates Host Inflammatory Response
}

\author{
Thomas Daniel Kraemer, ${ }^{1}$ Orlando Daniel Quintanar Haro, ${ }^{1,2}$ Eugen Domann, ${ }^{1}$ \\ Trinad Chakraborty, ${ }^{1}$ and Svetlin Tchatalbachev ${ }^{1}$ \\ ${ }^{1}$ Institute of Medical Microbiology, Justus Liebig University, Schubertstraße 81, 35392 Giessen, Germany \\ ${ }^{2}$ Iztacala Superior Studies Faculty, National Autonomous University of Mexico, Avenida de los Barrios 1, \\ Los Reyes Iztacala, 54090 Tlalnepantla, MEX, Mexico \\ Correspondence should be addressed to Svetlin Tchatalbachev; svetlin.tchatalbachev@mikrobio.med.uni-giessen.de
}

Received 3 April 2014; Revised 11 June 2014; Accepted 30 June 2014; Published 24 July 2014

Academic Editor: Michael McClelland

Copyright (c 2014 Thomas Daniel Kraemer et al. This is an open access article distributed under the Creative Commons Attribution License, which permits unrestricted use, distribution, and reproduction in any medium, provided the original work is properly cited.

Based on Toll/interleukin-1 receptor (TIR) domain structure homology, we detected a previously uncharacterized gene encoding for a TIR domain containing protein (Tcp) in the genome of Enterococcus faecalis. We assigned this gene the name $t c p F$ (as in Tcp of $E$. faecalis). Screening of $E$. faecalis samples revealed that $t c p F$ is more common in isolates from urinary tract infections (UTIs) than in human faecal flora. $t c p F$ alleles showed moderate single nucleotide polymorphism (SNP) among UTI isolates. Infection of mouse RAW264.7 macrophages with a tcpF knock-out mutant led to elevated cytokine response compared to the isogenic wild type E. faecalis strain. In silico analysis predicted significant tertiary structure homology to the TIR domain of human TLR1 (TLR1-TIR). When transiently expressed in cultured eukaryotic cells, TcpF caused suppression of TLR2-dependent NF- $\kappa$ B activation suggesting for TcpF a role as a factor in E. faecalis that benefits colonization by modulating the host's immune responses.

\section{Introduction}

Signaling via Toll-like receptors (TLRs) requires the homo- or heterodimerization of the receptors. The process is initiated by their extracellular leucine-rich repeats (LRRs) regions and leads to dimerization of the receptor's cytoplasmic Toll/interleukin-1 receptor (TIR) domains which form a TIRTIR structure [1]. This TIR-TIR structure provides the site of association with proteins of the TIR domain-containing adapter family [2,3]. Activation of TLR pathways leads to the release of inflammatory cytokines like tumor necrosis factor $\alpha$ (TNF- $\alpha$ ) and interleukin 6 (IL6), type I interferons (IFNs), and chemokines, which in turn control the recruitment of inflammatory cells to the infected tissues [4].

The central role of TLR signaling in innate immunity makes it a target for bacterial induced immune subversion. In 2006 the TIR-like protein, TlpA, from Salmonella enterica, was reported as a novel prokaryotic modulator of NF- $\kappa \mathrm{B}$ activity and interleukin 1 beta (IL-1 $\beta$ ) secretion that contributes to serovar Enteritidis virulence by interfering with TLR4 and MyD88 signaling [5]. Similar observations were also made with the TIR domain containing proteins TcpC of Escherichia coli and TcpB of Brucella melitensis [6]. TcpC was found to impair the signaling of TLRs and the secretion of proinflammatory cytokines IL6 and TNF- $\alpha$. Furthermore, $\operatorname{tcp} C$ was associated with the severity of urinary tract infections (UTIs) [6]. It was demonstrated that the TIR domain of TcpC was directly associated with MyD88 and TLR4 [7] and TcpB targeted the Toll/IL-1 receptor domain-containing adaptor protein (TIRAP) dependent pathway by mimicking TIRAP's affinity towards phosphatidylinositol phosphate (PIP) resulting in inhibition of TLR2- and TLR4-mediated signaling $[8,9]$. Another TIR domain containing protein Btpl from Brucella abortus downmodulates maturation of infected dendritic cells by interfering with the TLR2 signaling pathway [10]. Very recently a TcpC homolog protein (TirS) in 
Staphylococcus aureus was shown to attenuate TLR-induced activation of NF- $\kappa \mathrm{B}$ and MAP-kinase signaling pathways in vitro and contribute to organ colonisation upon $S$. aureus infection in vivo [11]. The mechanism of bacteria interfering with host TLR signaling by Tcps, by blocking endogenous protein's association, was described as molecular mimicry [12].

We detected the sequence for a TIR domain containing protein in the genome of Enterococcus faecalis strain Symbioflor 1 (GenBank: HF558530.1, Gene ID: EFS1_1683) $[13,14]$. The gene is also present in the published genomes of E. faecalis V583 (GenBank: AE016830.1, EF_1959) and Enterococcus sp. 7L76 draft genome (GenBank: FP929058). We assigned this gene the name $t c p F$, in accordance with the already described $t c p C$ and $t c p B$. In this work we analyze the distribution of $t c p F$ among clinical E. faecalis isolates, its genetic stability (allelic variation), the predicted protein structure, and its impact on the cellular innate response to infection. We report that $t c p F$ was more frequent in $E$. faecalis isolates from urinary tract infections (UTIs) than in human faecal flora isolates. A $t c p F$ deletion mutant of the model strain E. faecalis Symbioflor 1 induced elevated cytokine response upon infection of mouse macrophages compared to the parental wild-type strain and transient expression of TcpF in 293 human fibroblast cells led to suppression of the TLR2 triggered NF- $\kappa \mathrm{B}$ activation. Both findings indicate a role of TcpF in subversion of host defense by E. faecalis during infection.

\section{Materials and Methods}

2.1. Alignments and Protein Structure Calculation. Multiple protein sequence alignments were generated using Clustal Omega [15]. Protein tertiary structure prediction was conducted via HHpred by the Max-Planck Institute for Developmental Biology. Superposition of protein structures was done by TM-Align [16]. The structure of TLR1-TIR (1FYV) was downloaded from the protein data bank (http://www.rcsb.org/pdb/home/home.do). The presentations of the 3D TIR domain structures were accomplished by PyMOL software (http://www.pymol.org/).

2.2. Screening and Gene Sequencing. E. faecalis and E. coli isolation was performed from urine samples of patients with a clinically manifested UTI sent to the diagnostic unit of the Institute of Medical Microbiology, University Hospital Giessen and Marburg Ltd, branch Giessen. Colony PCR for tcps screening was done by picking one bacterial colony (ca. $10^{5}$ bacteria) and using the following primers: E. coli: UPECseq.1: 5' GAATGTTTTGGGCAACAATATG-3' and UPECseq.3: $5^{\prime}$-TCTTCTCCTGTATGCTATTTCAGC3'; E. faecalis: E.f.seq.1 and E.f.seq.2 (Table 1). PCR was carried out for 30 cycles $\left(\left(20 \mathrm{sec} \times 94^{\circ} \mathrm{C} / 30 \mathrm{sec} \times 55^{\circ} \mathrm{C} / 60 \mathrm{sec}\right.\right.$ $\left.\left.\times 72^{\circ} \mathrm{C}\right) \times 30 / 3 \mathrm{~min} \times 72^{\circ} \mathrm{C}\right)$ with initial denaturation of $10 \mathrm{~min} \times 94^{\circ} \mathrm{C}$. An aliquot of the PCR was resolved on $0.8 \%$ agarose gel. The positive isolates were subjected to a second PCR reaction in order to obtain DNA for sequencing using the following primers: for E. coli sequencing: UPECseq.6: $5^{\prime}$-GTGGAAAACCTTCTATGCC-3', UPECseq.7: $5^{\prime}$ GTGGTAGTTATCTATACCCTACATCTG-3', and for $E$. faecalis: E.f.seq.3 and E.f.seq.5 (Table 1). Resulting PCR fragments were purified using the QIAquick PCR purification Kit (Qiagen). Sequencing was done by AGOWA genomics, Berlin (http://www.agowa.de/). The sequencing results were analyzed with SeqManII (DNAStar) software. A second set of $31 \mathrm{E}$. faecalis isolates was collected from stool samples of non-UTI patients, where E. faecalis was determined to be not the cause of the illness, and screened for the presence of $t c p F$ as described above. Statistical analysis for the significance of $t c p F$ distribution among isolates was performed using Chisquared test [6].

2.3. Bacterial Strains. E. coli CFT073 (NC004431) was a kind gift from U. Dobrindt, Institut für Molekulare Infektionsbiologie, JMU Würzburg, Germany. E. faecalis Symbioflor 1 (DSM 16431) is a commercially available probiotic strain and was provided by K. Zimmermann, SymbioPharm, Herborn, Germany [13]. E. faecalis V583 was donated by J. Hübner, Division of Infectious Diseases, University Hospital Freiburg, Germany. E. coli CFT073 (UPEC) was grown on Columbia agar (Oxoid) or Luria-Bertani (LB) broth at $37^{\circ} \mathrm{C}$. E. faecalis was grown in brain-heart-infusion (BHI) broth or on $\mathrm{BHI}$ agar plates (Difco) at $37^{\circ} \mathrm{C}$. E. coli TOP 10 (Invitrogen) was grown in Luria-Bertani (LB) broth at $37^{\circ} \mathrm{C}$ on $\mathrm{LB}$ agar plates (Difco). If required, antibiotics were added with the following concentrations: ampicillin: $100 \mu \mathrm{g} / \mathrm{mL}$, kanamycin: $50 \mu \mathrm{g} / \mathrm{mL}$, and erythromycin: $10 \mu \mathrm{g} / \mathrm{mL}$ for E. faecalis and $300 \mu \mathrm{g} / \mathrm{mL}$ for E. coli. For infection assays E. faecalis were grown in BHI broth to early exponential phase $\left(\mathrm{OD}_{600} 0.1-\right.$ 0.5 ) and the bacterial concentration was calculated by optical density from a standardized growth curve.

2.4. DNA Isolation and Manipulation. Genomic DNA from E. coli and E. faecalis was isolated using the Genomic DNA purification kit (Gentra). Plasmid preparations from E. coli TOP 10 were done using the QIAfilter Plasmid Midi Kit (Qiagen). Knock-out of the $t c p F$ gene in the genome of $E$. faecalis Symbioflorl was done as described [17]. Briefly, the flanking regions of the $t c p F$ gene were amplified by PCR (Expand High fidelity PCR system, Roche) using the primer pairs Ef-Del-1A 5'-AGCACTGGATCCGAACCAATTCTCCTTATACTCG-3' and Ef-Del-2 $5^{\prime}$-GACGACTACACAGTGCTCACTACTCAACATCCTCCA-3', giving rise to a 345-bp product I, Ef-Del-3 5'-ACTGTGTAGTCGTCGGTAGAGTAATTGGAGGAGGT-3', and Ef-Del-4 5'-AGCAGTGAATTCAATGCAGCTGTTTCTAACAACCGA-3', generating a 405-bp product II. A second PCR was performed with primers Ef-Del-1A and Ef-Del-4 using both products I and II as a template to amplify a 736-bp DNA fragment, which was subsequently digested (Bam HI and EcoRI), ligated into the temperature sensitive shuttle vector pAULA (25), and transformed into $E$. coli TOP10. The vector construct was isolated, sequenced, and electroporated into E. faecalis to achieve gene deletion. 
TABLE 1: RT and PCR primer for Enterococcus faecalis.

\begin{tabular}{|c|c|c|}
\hline Primer & Sequence & $5^{\prime}$ position \\
\hline E.f.seq.1 & $5^{\prime}$-GATGTTGAGTAGTGAGCAACG-3' & $-12 \mathrm{~s}$ \\
\hline E.f.seq. 2 & $5^{\prime}$-СТССТССААТТАСТСТАССТТСТС-3' & 832 as \\
\hline E.f.seq.3 & 5'-CCGTACATCGCTCTATAATCAC-3' & $-203 s$ \\
\hline E.f.seq. 5 & $5^{\prime}$-GTCTAAATGCATCACTTTTAATTACTC-3' & 995 as \\
\hline E.f.seq. 6 & 5'-AGCCATTCCAAAATAAACTGAC-3' & $470 \mathrm{~s}$ \\
\hline E.f.seq.7 & 5'-GAATTCCCGATTGTTATACG-3' & 770 as \\
\hline E.f.seq. 8 & 5'-GCTTTCATCCTCTGATAGCGT-3' & 606 as \\
\hline E.f.seq.14 & 5'-AGTCATAGTTCAAAGGATCAAG-3' & $29 \mathrm{~s}$ \\
\hline E.f.seq.15 & 5'-CTGATCATTATTATCAGAGTATTCCATG-3' & $212 \mathrm{~s}$ \\
\hline E.f.seq.17a & 5'-GTAGTGAGCAACGGGAAAAAGAT-3' & $-3 s$ \\
\hline
\end{tabular}

s: sense.

as: antisense.

$T c p F$ and $t c p C$ were cloned into the eukaryotic expression plasmid pCMV-Flag-2B (Stratagene), generating the plasmids pSty10 (TcpF) and pSty13 (TcpC1). The following primer combinations were used to amplify genomic DNA for the cloning: TK9: 5'-ACTACTGGTCTCAGCGCCAATGAGAGTATCAGT- $3^{\prime}$ and TK10: $5^{\prime}$-ACGACTGGTCTCATATCAGATAAATAATTTAACTGGCT- $3^{\prime}$ for E. faecalis TcpF (pSty10); pTcpC-Flag For: $5^{\prime}$-ATCCGCGGATCCATAGCATATGAAAACATAGAATTT-3' and pTcpC-Flag Rev: $5^{\prime}$ GCGAGTCTCGAGTTATCTTCTCCTGTATGCTATTTCAGC-3' [6] for full length $E$. coli tcpC (TcpC1) originally a GTG start codon (pSty13). The vector and the amplified DNA fragments were cut with BamHI and XhoI (Fermentas) in order to prepare compatible cohesive ends for ligation.

2.5. RNA Isolation and RT-PCR. For RNA isolation, E. faecalis Symbioflor 1 and V583 were harvested after growth in BHI broth or from the cell culture supernatants (DMEM) of CaCo cells after 1, 2, or $3 \mathrm{~h}$ of infection. Total RNA was isolated using the RNeasy Protect Bacteria Mini Kit (Qiagen) following the manufacturers' protocol with lysozyme lysis. The RNA was eluted in RNase free water, digested with $2 \mathrm{U}$ of Turbo DNaseI (Ambion) for $30 \mathrm{~min}$ at $37^{\circ} \mathrm{C}$, and again purified using the RNeasy Mini Kit (Qiagen). RNA was recovered in RNase free water, heat denatured for $10 \mathrm{~min}$ at $65^{\circ} \mathrm{C}$, and quantified with the NanoDrop ND-1000 UVVis Spectrophotometer (NanoDrop Technologies), and a quality profile with the Agilent 2100 bioanalyzer (Agilent Technologies) was made. For RT-PCR, $400 \mathrm{ng}$ total RNA was reverse transcribed using $50 \mathrm{U}$ Superscript II Reverse Transcriptase (Life Technologies) in a $20 \mu \mathrm{L}$ reaction using primers E.f.seq. 7 or E.f.seq. 2 (Table 1) following the instructions of the manufacturer. PCR was done with $3 \mu \mathrm{L}$ of first strand cDNA in a $30 \mu \mathrm{L}$ reaction using $1 \mathrm{U}$ Taq Polymerase (Life Technologies). PCR was carried out for 30 cycles ((20 sec $\left.\left.\times 94^{\circ} \mathrm{C} / 30 \mathrm{sec} \times 53-56^{\circ} \mathrm{C} / 60 \mathrm{sec} \times 72^{\circ} \mathrm{C}\right) \times 30 / 3 \mathrm{~min} \times 72^{\circ} \mathrm{C}\right)$. $8 \mu \mathrm{L}$ of the PCR reaction was resolved on $1.5 \%$ agarose gels.

2.6. Luciferase Reporter Assay. Human TLR2 and luciferase expressing plasmids are described previously [18]. Confluent overnight cultures of HEK 293 cells in 12-well plates, grown in DMEM (Gibco) supplemented with $10 \%$ foetal calf serum (PAA Laboratories) and 1x penicillin/streptomycin (Gibco), were washed in DMEM and transfected with a mixture of $2.4 \mu \mathrm{g}$ plasmid DNA and $7 \mu \mathrm{L}$ Lipofectamine 2000 (Invitrogen). All assays were performed with a constant amount of reporter constructs as follows: $550 \mathrm{ng}$ pELAM-Luc and $50 \mathrm{ng}$ phRL-TK (Promega), $50 \mathrm{ng}$ phTLR2, and increasing amounts of TcpC1 (50 ng to $1550 \mathrm{ng}$ of pSty13) or TcpF (50 ng to $1550 \mathrm{ng}$ of pSty10). DNA to Lipofectamine ratio was kept stable by using the empty CMV-promoter vector pRK5 (BD Pharmingen). After $6 \mathrm{~h}$ the medium was replaced with DMEM supplemented with 10\% FCS and after an additional $2 \mathrm{~h}$, the cells were split, transferred to 96 -well plates at $4 \times$ $10 \%$ well, and incubated overnight in DMEM supplemented with $5 \%$ FCS. On the next day the cells were stimulated with $0.5 \mu \mathrm{M} \mathrm{Pam}{ }_{3}$ Cys-SKKKK $\times 3 \mathrm{HCl}$ (EMC microcollections $\mathrm{GmbH}$, Tübingen) in DMEM. Control samples were treated with an equal volume of DMEM. Stimulated cells were incubated for $5 \mathrm{~h}$, lysed afterwards with $20 \mu \mathrm{L}$ Passive Lysis Buffer (Promega), and stored at $-20^{\circ} \mathrm{C}$. Measurement of NF$\kappa \mathrm{B}$ mediated luciferase activity was carried out with the DualLuciferase Reporter Assay System (Promega) using an L-max plate reader (Molecular Devices). Signal measurements were performed in triplicate and experiments were repeated at least three times. The primary firefly luciferase signal was normalized to the signal of Renilla luciferase in each well, resulting in relative luciferase activity. Results are presented as fold changes of stimulated to nonstimulated activity.

2.7. Cytokine Assays. RAW 264.7 mouse macrophages were seeded on 24 -well plates at $6 \times 10^{5}$ cells/well in $500 \mu \mathrm{L} \mathrm{DMEM}$ supplemented with $10 \%$ FCS and $1 \mathrm{x}$ penicillin/streptomycin. On the next day the cells were washed twice with PBS $\left(37^{\circ} \mathrm{C}\right)$ and covered with $400 \mu \mathrm{L}$ DMEM + 1\% FCS. The RAW 264.7 cells of two control wells were harvested and counted in order to define exact cell numbers. $100 \mu \mathrm{L}$ bacterial suspension in DMEM, containing a defined CFU number, was added to the cells and incubated for $5 \mathrm{~h}$. Supernatants were collected from cells infected with $E$. faecalis Symbioflor 1 and the isogenic $\Delta t c p F$ mutant. The secretion of TNF- $\alpha$ was measured using the mouse TNF- $\alpha$ Quantikine ELISA kit (BD) on a Phomo 
Plate reader (Anthos Mikrosystme) according to the kits manual.

\section{Results}

3.1. In Silico Modeling of TcpF. In a database search of bacterial genomes using conserved domain database (CDD) on NCBI, we identified a gene encoding for a TIR domain containing protein in the genome of E. faecalis strain Symbioflor 1 (Gene ID: EFS1_1683). The sequence spans 825 base pairs coding for a 274-amino-acid-long protein with predicted mass of $32650 \mathrm{Da}$. Concordant with previous studies [6], we assigned this gene the name TIR domain containing protein of E. faecalis $(t c p F)$. Additionally, a multiple primary sequence alignment of the human TIR domain containing proteins involved in TLR2 signaling, TLR1, TLR2, MyD88, and MAL, and the bacterial TIR domain containing proteins PdTLP, TcpC, and $\mathrm{TcpF}$ [12] revealed strong consensus in the Boxl motif [19], the last two residues, proline $(\mathrm{P})$ and glycine $(\mathrm{G})$, of the Box2 motif, and the residues forming the $\beta \mathrm{C}$-strand and the $\alpha \mathrm{C}$ helix (Supplementary Figure 1 in Supplementary Material available online at http://dx.doi.org/10.1155/2014/918143). The primary sequence of TcpF-TIR exhibits common TIR domain motifs. The predicted tertiary structure of TcpF-TIR shows a typical TIR domain fold with parallel $\beta$-strands surrounded by $\alpha$-helices and loops (Supplementary Figure 2(a) In silico structure prediction of TcpF-TIR reveals structural similarity to TLR1-TIR.). The TIR domain of TcpF is located at the Nterminus and consists of $4 \beta$-strands, which are coated by $4 \alpha$-helices. The superposition of TcpF-TIR and TLR1-TIR reveals considerable similarity between those two structures (Supplementary Figure 2(b)). In particular, strong similarity can be observed at the BB-loop, which has been reported to be part of the interface of TLR1-TIR and TLR2-TIR interaction (Supplementary Figure 2(c)) [20]. Also located at the Cterminus of TcpF, we found the motif KVRFKLKK similar to a functional motif in TcpB of Brucella, a PIP2 binding domain with the basic amino acid sequence KKRxxxxKK. It was reported that this motif promotes colocalization with the plasma membrane of the eukaryotic cell by binding to membrane phosphoinositides [9].

3.2. Screening of E. faecalis Strains for tcpF. A total of 110 urine isolates of patients with clinically diagnosed UTI and 31 faecal flora $E$. faecalis isolates were screened via PCR with primers E.f.seq. 1 and E.f.seq. 2 positioned at the start and the end of the $t c p F$ open reading frame (ORF). The UTI isolates were collected in a period of three months from samples sent to the microbiology diagnostic lab of the Institute of Medical Microbiology. The samples originate from patients admitted to the University Hospital in Giessen with clinically apparent UTI. Among UTI samples, E. faecalis colony-forming unit (CFU) numbers varied with respect to the threshold of $<10^{5} / \mathrm{mL}$ and $>10^{5} / \mathrm{mL}$; the latter fulfill the microbiological definition of UTI. E. faecalis stool isolates were derived from stool samples of non-UTI patients, where E. faecalis was determined as not the causative agent, but element of the stool commensal flora. TcpF was found in $76.4 \%$ of all UTI isolates.

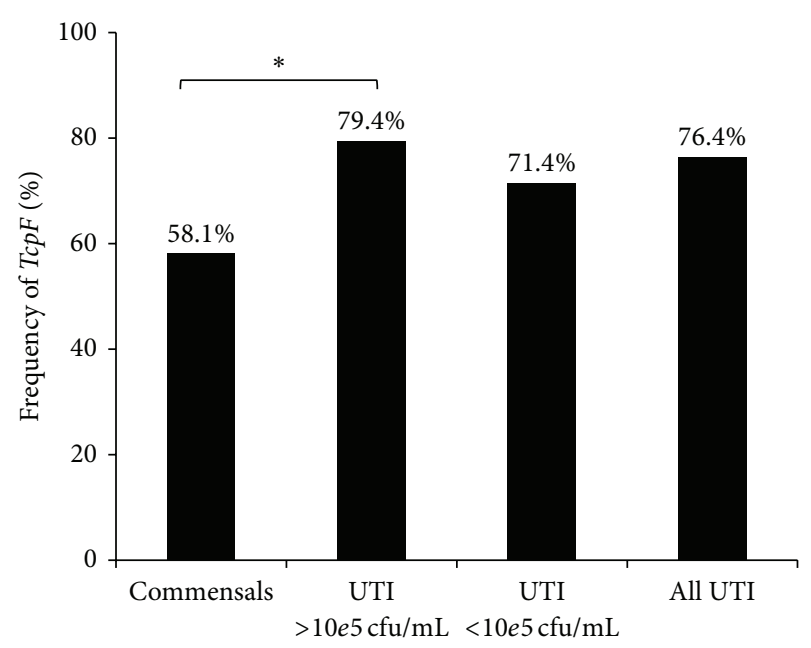

FIGURE 1: TcpF frequency among UTI and commensal isolates. TcpF is associated with $\mathrm{cfu}>10 \mathrm{e} 5 / \mathrm{mL}$ upon infection of the urinary tract by $E$. faecalis. TcpF was detected by PCR. Commensals $n=31$, UTI $>10 \mathrm{e} 5 \mathrm{cfu} / \mathrm{mL}, n=68\left({ }^{*} \mathrm{P}=0,028\right)$, UTI $<10 \mathrm{e} 5 \mathrm{cfu} / \mathrm{mL} n=42$. Statistical analysis was performed using Chi-squared test.

Interestingly, tcpF was present in $79.4 \%$ of UTI isolates from samples with CFU numbers $>10^{5} / \mathrm{mL}$ but was less common in UTI isolates from samples with CFU numbers < $10^{5} / \mathrm{mL}(71.4 \%)$ or isolates from faecal flora (58.1\%) (Figure 1). These numbers suggest that the presence of $t c p F$ benefits the colonization of the human urinary tract (UT) and promotes E. faecalis survival.

3.3. Genetic Analysis of tcpF. We further examined the genetic stability of $t c p F$ among urine isolates by sequencing the target gene and the neighboring regions. A PCR with primers E.f.seq. 3 and E.f.seq. 5 was done for a total of 68 isolates including isolates from urine samples with more than $10^{5} \mathrm{CFU} / \mathrm{mL}(n=44)$ and less than $10^{5} \mathrm{CFU} / \mathrm{mL}$ $(n=22)$. The primers are located about $150-200 \mathrm{bp}$ up- and downstream from the $t c p F$ gene. The amplified PCR fragment has an expected length of 1206 bp. Primers E.f.seq. 3 and E.f.seq. 5 were reused for bidirectional sequencing. The SNPs aligned to the $t c p F$ sequence of E. faecalis Symbioflor 1 are shown in Figure 2(a). In total, we identified $18 t c p F$ genotypes and found four nucleotide substitutions leading to amino acid changes. The transition G121A was the most frequent (85.3\%); thus it seems to be the more stable variant. This SNP is located within the TIR domain and leads to the change of alanine to threonine. The transversion $\mathrm{C} 226 \mathrm{G}$ is located also within the TIR domain resulting in a glutamine to lysine exchange. Only one strain showed this substitution. The transitions C551T and T809C are outside of the TIR domain. C551T results in a threonine to isoleucine exchange and was found in 40 tcpF positive isolates (58.8\%). T809C was found in one isolate and results in leucine to serine exchange. The G121A and C551Talleles were randomly distributed among the isolates from urine samples with CFU $>10^{5} / \mathrm{mL}$ and CFU $<10^{5} / \mathrm{mL}$.

Two E. faecalis urine isolates produced PCR fragments of unexpected length suggesting a larger insertion within 


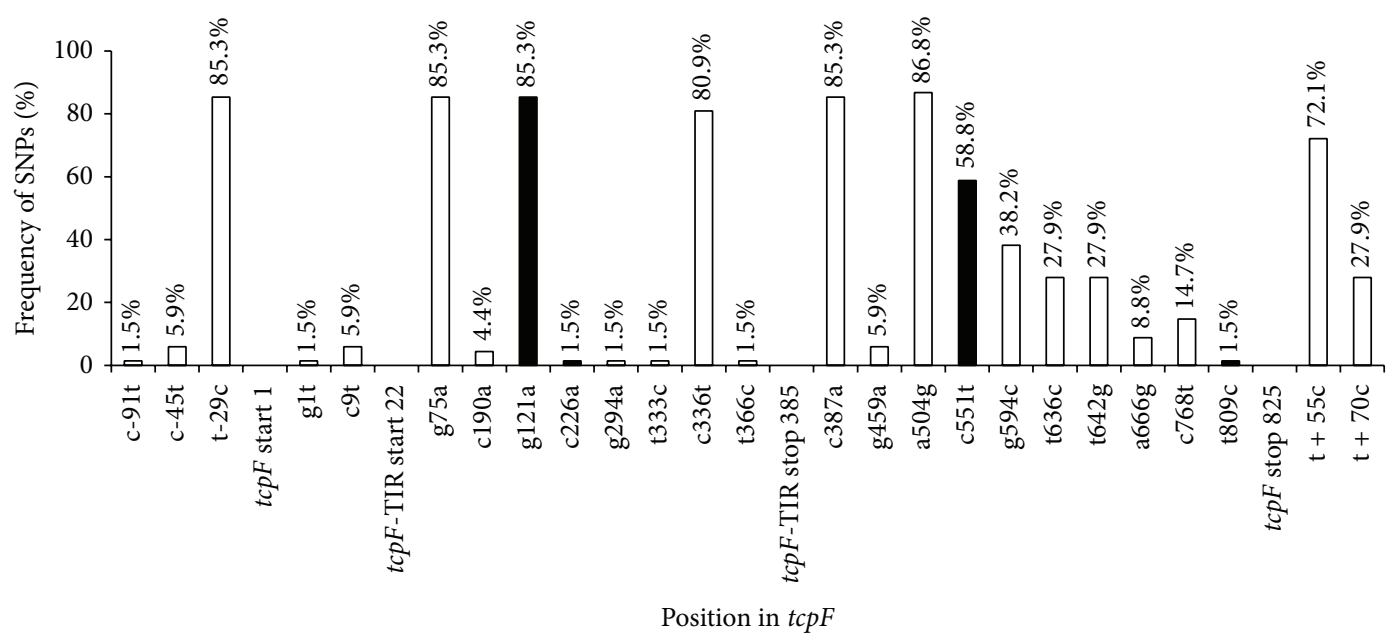

(a)

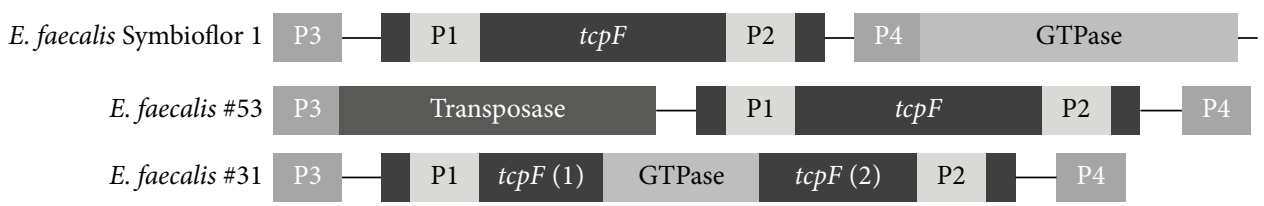

(b)

FIgURE 2: TcpF's sequence polymorphism. (a) SNP frequency in the $t c p F$ locus of 68 E. faecalis UTI isolates. Sequence alignment was conducted on $t c p F$ of $E$. faecalis Symbioflor 1. Black bars indicate mutations resulting in amino acid substitutions and white bars indicate silent mutations. The amino acid substitutions are G121A: Ala-Thr, C226G: Gln-Lys, C551T: Thr-Ile, and T809C: Leu-Ser. (b) Genetic structure of the $t c p F$ locus of $E$. faecalis isolates. Two major rearrangements were observed in the UTI isolates E. faecalis \#53 and \#31. P1/P2: primer positions for the screening-PCR; P3/P4: primer positions for the sequencing-PCR.

the primers' positions (Figure 2(b)). Isolate E.f.\#53 yielded the expected fragment of $844 \mathrm{bp}$ in the screening PCR. However, the sequencing PCR produced a fragment of about $2000 \mathrm{bp}$ by expected $1206 \mathrm{bp}$. The sequencing revealed a $5^{\prime}$ end insertion followed by the open reading frame of $t c p F$. The search in the genome of E. faecalis Symbioflor 1 showed this inserted sequence to encode for a gene with annotated transposase function (EFS1_1430). Isolate E.f.\#31 produced a fragment of unexpected length in the screening as well as in the sequencing PCR. The amplified fragment of the screening PCR was about $1800 \mathrm{bp}$ and the fragment of the sequencing PCR was about $2100 \mathrm{bp}$, revealing that the ORF of $t c p F$ was disrupted by an inserted fragment. A genomic BLAST search identified a homolog sequence in the genome of E. faecalis V538 encoding for the gene downstream of $t c p F$ with annotated putative deoxyguanosine triphosphate triphosphohydrolase (GTPase) function (EF_1958). In both, the genomes of E. faecalis Symbioflor 1 and V538, the putative GTPase gene starts in close proximity (17 bp) to the $3^{\prime}$ end of tcpF.

In order to compare $t c p F$ 's genetic variation to other bacterial tcps we screened $100 \mathrm{E}$. coli isolates derived from urine specimens of UTI patients for the presence of the tcpC sequence. All isolates originated from samples with CFU numbers $>10^{5} / \mathrm{mL}$. 20 E. coli isolates (20\%) were $t c p C$ positive. Unlike $t c p F$, sequencing of $t c p C$ revealed very low genetic polymorphism. The sequence of 19 isolates was identical to CFT073's tcpC. One isolate only showed two point mutations. The transition G385A led to an alanine to valine exchange, whereas the transition C482T was silent.

The transcription of $t c p F$ mRNA was verified for $E$. faecalis Symbioflor 1 (Figure 3(a)) as well as for the pathogenic isolate V583 (data not shown). cDNA transcripts with increasing length were observed using reverse transcription primer E.f.seq. 2 positioned between base pairs 809 and 832 (stop codon TAA at $823-825 \mathrm{bp}$ ), and PCR with primers which span the length of the gene up to the start codon. TcpF was transcribed during growth of bacteria in BHI medium and also by coincubation (infection) with colon carcinoma CaCo-2 cells for up to three hours (Figure 3(b)).

3.4. Functional Analysis of TcpF. In order to analyze the function of TcpF during infection, we created a $t c p F$ deletion mutant of the model strain E. faecalis Symbioflor $1=$ Symbioflor $1 \Delta t c p F$. The deletion did not affect bacterial growth in BHI medium (Figure 4(a)), but infection of mouse RAW 264.7 macrophages with the deletion mutant led to significantly higher TNF- $\alpha$ secretion in medium than that caused by the wild-type strain Symbioflor 1 (Figure 4(b)), revealing inhibition of stress signaling by TcpF. Further studies should include infection experiments of mouse macrophages with a complemented strain (E. faecalis Symbioflor $\Delta t c p F+t c p F$ ) in order to additionally support and verify the knock-out 


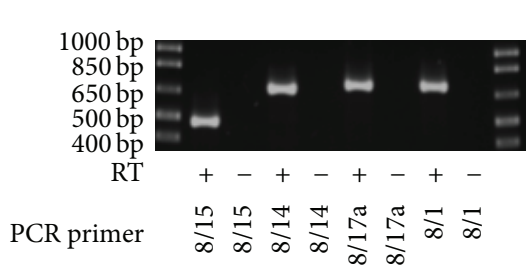

(a)

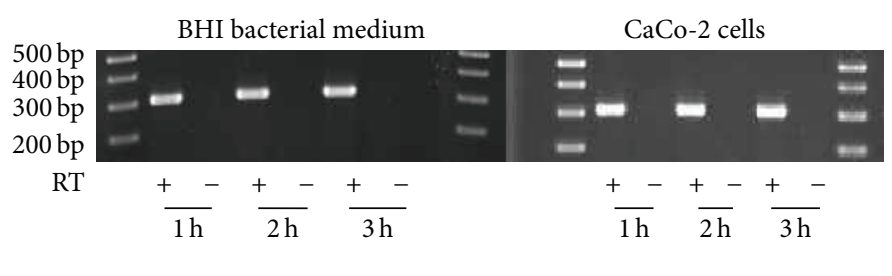

(b)

Figure 3: TcpF is constitutively transcribed in E. faecalis Symbioflor 1. (a) Full length mRNA transcript of $t c p F$ in E. faecalis Symbioflor 1. Reverse transcription was performed with primer E.f.seq.2. PCR primers E.f.seq. 8/15: 457 bp, primers E.f.seq. 8/14: 640 bp, primers E.f.seq. 8/17a: 671 bp, and primers E.f.seq. 8/1: 680 bp. (b) TcpF mRNA is transcribed upon growth in BHI medium and upon infection on CaCo cells. PCR primers E.f.seq. 7/6: 300 bp. Reverse transcriptase minus (RT-) reactions served as negative control.

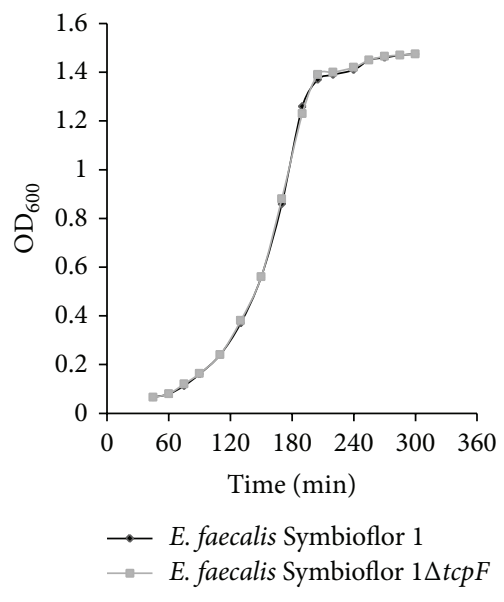

(a)

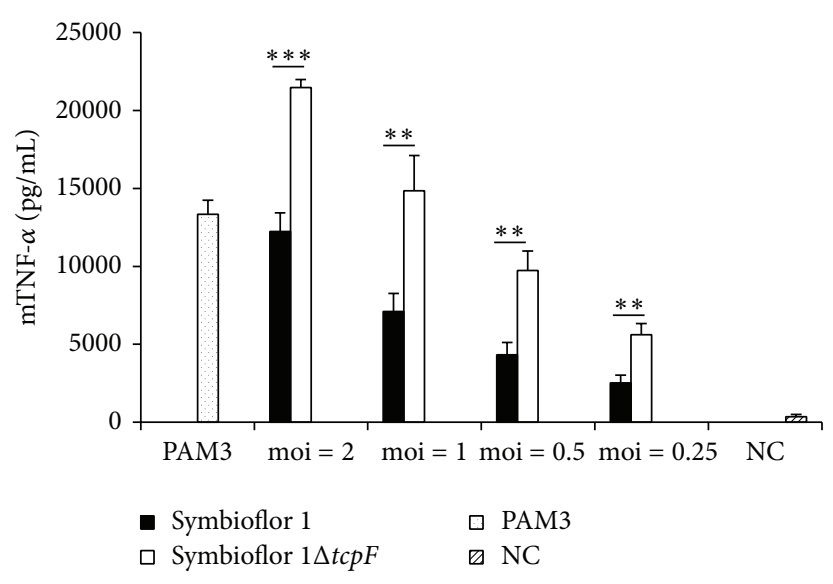

(b)

Figure 4: TcpF suppresses TNF- $\alpha$ induction. (a) E. faecalis Symbioflor 1 and its isogenic tcpF deletion mutant Symbioflor $1 \Delta t c p F$ show identical growth rate. Fresh culture was started from overnight culture $1: 25 \mathrm{in} \mathrm{BHI}$ at $37^{\circ} \mathrm{C}$ and $100 \mathrm{rpm}$. Optical density at $600 \mathrm{~nm}\left(\mathrm{OD}_{600}\right)$ at indicated time points. (b) RAW264.7 macrophages were infected for $6 \mathrm{~h}$ with E. faecalis Symbioflor 1 and $E$. faecalis Symbioflor $1 \Delta t c p F$ at indicated moi. NC: negative control—uninfected macrophages. Error bars represent s.d. $(n=4)$. Student's $t$-test: ${ }^{* * *} P<0,001$; ${ }^{* *} P<0,01$.

phenotype and gene function. Previous studies have shown that immune suppression by bacterial Tcps is based on direct interaction with host's Tcps leading to interference with TLR signaling $[6,7,11]$. TLR2 is responsible for sensing Gram-positive pathogens [18]; hence we expressed cloned TcpF and TcpC in human fibroblast cells and monitored the effect on the TLR2 dependent NF- $\kappa$ B activation. Therefore, HEK293 cells were transiently transfected with plasmids expressing the firefly luciferase gene under the control of an NF- $\kappa \mathrm{B}$ dependent promoter (pELAM-Luc) and plasmids constitutively expressing human TLR2 (phTLR2). Plasmids for constitutive eukaryotic expression of Tcps : pSty10 (TcpF) or pSty13 (TcpC of UPEC CFT073) were cotransfected in a ratio of $1: 1,1: 2,1: 5,1: 10$, and $1: 31$ to phTLR2. Transfected cells were stimulated with $\mathrm{Pam}_{3}$, a synthetic ligand of TLR2. Intracellular expression of TcpF resulted in dose dependent NF- $\kappa \mathrm{B}$ signal suppression comparable to that of TcpC (Figure 5). These results suggest that TcpF interferes with TLR signaling in a similar manner to TcpC [6] resulting in markedly reduced cytokine release and impaired response upon infection.

\section{Discussion}

Over the last decade several bacterial TIR domain-containing proteins (Tcps) have been identified [21], yet all but one were found in Gram-negative bacteria [11]. Using a conserved protein domain search we discovered a homologous sequence for the TIR domain encoded by a gene of previously unknown function in the genome of E. faecalis (E. faecalis V583: EF_1959; E. faecalis Symbioflor 1: EFS1_1683) [13, 14]. Enterococci have until recently been considered harmless or even beneficial commensals of the gastrointestinal tract, the oral cavity, and vagina in humans. However, there is growing evidence that these bacteria possess specific traits that enable them to colonize new habitats in the host and cause infections such as bacteremia, peritonitis, endocarditis, device-related infections, and urinary tract and wound infections [22]. Enterococci, and particularly the species E. faecalis, are second to E. coli to cause UTIs [23].

In a screening of UTI and stool isolates, we found that the TIR containing gene, $t c p F$, is less frequent in commensal E. faecalis strains (58.1\%) than in isolates from UTI $(76.4 \%)$ 


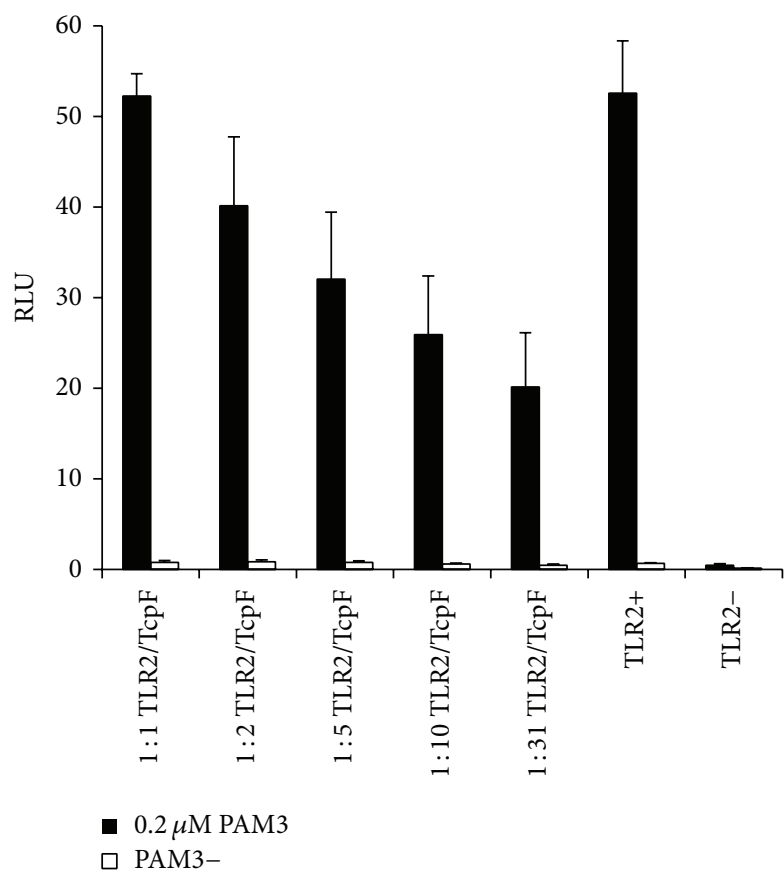

(a)

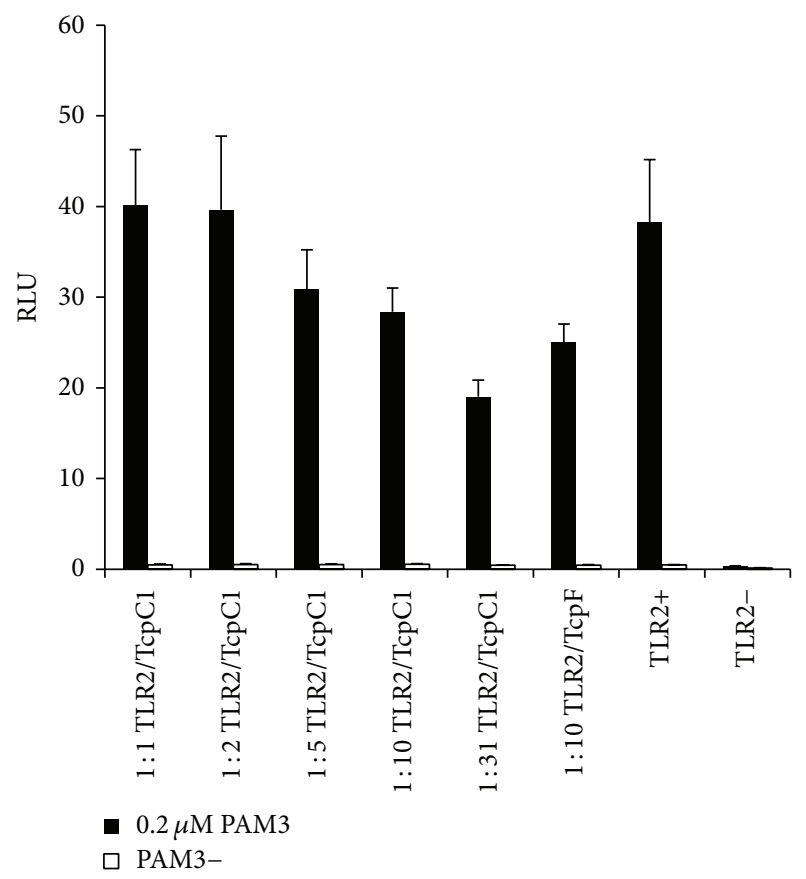

(b)

FIGURE 5: Intracellular expression of TcpF impairs TLR2 dependent NF $\kappa$ B activation. HEK293 cells were transiently transfected with hTLR2 (50 ng), pRK5, reporter plasmids pELAM-Luc and phRL-TK, and increasing amounts of TcpF (a) or TcpC1 (b) expressing plasmids at ratios of $1: 1,1: 2,1: 5,1: 10$, and $1: 31$. Cells were stimulated with $0.2 \mu \mathrm{M}$ PAM3 and incubated for $5 \mathrm{~h}$. Nonstimulated and TLR2 deficient cells (TLR2-) served as controls. Error bars represent s.d. $(n=3)$. RLU: relative luciferase unit.

and this difference becomes more significant when only the UTI isolates with cfu numbers higher than $10 \mathrm{e} 5 / \mathrm{mL}(79.4 \%$ tcpF positive) are compared with the commensals $(P=$ 0.028 ) (Figure 1), suggesting that the $t c p F$ promotes bacterial survival and therefore constitutes a selective advantage for the colonization of the human UT by E. faecalis. Similar observations were made by Cirl et al. 2008 [6] regarding the TIR domain containing protein of $E$. coli (TcpC). They reported that TcpC promotes intracellular survival in immune and epithelial cells and that $t c p C$ was associated with severity of UTI in humans. In their study tcpC was found in $40 \%$ of acute pyelonephritis isolates but was less common in cystitis (21\%), asymptomatic bacteriuria (16\%), or commensal $(8 \%)$ E. coli strains. Interestingly, we found $t c p F$ to be also abundant (58.1\%) among commensal (nonpathogenic) isolates from faecal flora, indicating a stronger beneficial effect of the Tcp gene for E. faecalis than that for E. coli (8\%) in the colonization of the human GI tract.

The sequence analysis of $t c p C$ revealed just one point mutation with a subsequent amino acid change and one silent point mutation among $20 t c p C$ positive $E$. coli isolates. Both nucleotide substitutions do not concern the TIR domain. However, in the $825 \mathrm{bp}$ long sequence of $t c p F$ we detected point mutations in 20 positions and 4 of them resulted in subsequent amino acid changes. Two of them are within the TIR domain, yet one of them C226G is of very low frequency ( 1 in 68). Thus, in contrast to $t c p C, t c p F$ shows a moderate genetic polymorphism, which however translates only to a lower extent in primary amino acid sequence variation. In silico modeling of the TIR domain of TcpF reveals a conserved (stable) amino acid sequence resulting in conserved secondary and tertiary structures (Supplementary Figure 2(a)). The observed alteration Ala41Thr leads to structural changes in the beginning of the BB-loop (Supplementary Figure 2(d)), whereas the alteration Gln76Lys does not influence the TIR domain structure (Supplementary Figure 2(e)). Whether Ala41Thr affects binding towards possible binding partners is a matter of speculation, which requires further experiments, for example, TLR2 coexpression, to provide a definite answer. Remarkably, we observed also two major insertions in this gene locus among the isolated strains. In the case of E.f.\#53 a transposition led to alteration of the neighboring sequence of $t c p F$. In the case of E.f. $\# 31$, an insertion of the downstream positioned GTPase gene into the $t c p F$ ORF took place, most probably the result of an inversion (Figure 2(b)). It appears that the GTPase (EFS1_1682; EF_1958) was part of the insertion loop and was rearranged into the $t c p F$ gene, based on the known insertion mechanisms [24]. Both locus rearrangements and also the higher number of single nucleotide polymorphisms in $t c p F$ compared to $t c p C$ support the hypothesis of a process of ongoing selection and a more recent acquisition of the sequence in the genome of $E$. faecalis. Interestingly, in all $t c p F$ positive isolates $t c p F$ is always paired with the downstream positioned putative GTPase gene. However, no functional dependency or interrelationship between both genes has been 
reported yet or was predicted when comparing homologous protein domains in other bacteria. Both genes are not part of any known cluster being positioned $350 \mathrm{bp}$ away from the next upstream and $630 \mathrm{bp}$ from the next downstream neighbor.

Bacterial Tcps have been postulated to modulate host immune response by interference with the TLR signaling pathway $[11,12]$. Using the mouse macrophage line RAW264.7 to investigate the effect of TcpF on host immune cells, we found that infection with the tcpF deletion mutant of E. faecalis strainSymbioflor 1 led to significantly higher TNF- $\alpha$ response as compared to wild-type (Figure 4(b)). TNF- $\alpha$ promotes the acute phase reaction and leads to the recruitment of inflammatory cells [4]. Our results show that TcpF enables E. faecalis to attenuate host's cytokine response upon infection to avoid elimination by immune cells. Finally, the ability to suppress the TLR 2 dependent NF- $\kappa$ B activation for TcpF was shown to be similar to the suppression caused by TcpC as demonstrated by Cirl et al. 2008 [6]. TLR2's main ligands are bacterial lipoproteins allowing the receptor to react by stimuli from both Gram-positive and Gram-negative bacteria [3]. The intracellular expression of TcpF suppressed the TLR2 dependent NF- $\kappa$ B signal in a dose dependent manner (Figure 5). Considering molecular mimicry of human TIR domains as the mechanism for the immune modulatory effect of bacterial TIR containing domain proteins, TcpF's possible targets in the TLR2 dependent pathway are the TIR domains of TLR2, TLR1, TLR6, and MyD88 as well as MAL [4]. The structure prediction revealed that TcpF-TIR shows high similarity to TLR1-TIR. Since the BB-loop of TLR1-TIR and in particular the Gly 49 within the BB-loop (Supplementary Figure 2(c)) were shown to be involved in TLR1-TIR and TLR2-TIR interaction [20], it seems likely that TcpF may compete with the TIR domain of human TLR1 for TLR dimer formation and so abolishes further downstream signaling. In this context a colocalization of TcpF with the cytoplasmic part of TLRs may be facilitated by the presence of the KVRFKLKK motif at the C-terminus of TcpF as reported for the KKRxxxxKK motif in TcpB of Brucella [9].

In spite of their pathogenic potential, enterococci are exemplary commensals as evidenced by their presence as natural colonizers of the GI tract and by the fact that they have been used safely for decades as probiotics [13, 25]. Avoiding activation of $\mathrm{NF}-\kappa \mathrm{B}$ via TLR inhibition is crucial for homeostasis in the GI tract, where epithelial cells are in constant contact with the commensal microbiota. Accordingly, inhibitory molecules that minimize the risk of perpetual activation, such as the TLR inhibitors PPARy, A20, NOD2, IRAK-M, SIGIRR, and Tollip, are essential for sustaining mucosal homeostasis in the human gut [26]. Inappropriate activation of TLR signaling pathways leads to deleterious inflammation and has been implicated in the pathogenesis of GI disorders, including inflammatory bowel disease [27] and colon cancer [28]. On account of the higher frequency of polymorphisms detected in the tcpF ORF, this gene's acquisition appears to be a relatively recent event for the genome of E. faecalis. This gene seems to be beneficial for the colonization of the UTI tract by Enterococcus faecalis since $79 \%$ of UTI isolates with CFU numbers $>10^{5} / \mathrm{mL}$ carry this gene and even $71.4 \%$ of the UTI isolates with CFU numbers $<10^{5} / \mathrm{mL}$ are also positive for the gene. On the other hand, the gene is also present in more than the half of commensal isolates from stool samples; thus this recently acquired gene is spreading successfully among commensal E. faecalis strains, also indicating a positive effect for the colonizing bacteria in the gut. In both the pathogenic and commensal strains, the presence of $t c p F$ serves the paradigm of attenuating TLR2 signaling, therefore limiting bacterial recognition and immune activation by the host and therefore enabling colonizing bacteria to reach higher cfu numbers. It appears that TcpF promotes E. faecalis invasion and survival in the UTI and while in its natural polymicrobial habitat, the human GI tract, TcpF benefits the intestinal homeostasis.

\section{Conclusion}

In this work we provide evidence that TcpF from E. faecalis is a new member of the family of bacterial Tcps in the phylum of Gram-positive bacteria. In silico structure prediction of TcpFTIR showed a characteristic TIR domain fold and strong similarity to TLR1-TIR in particular. A screening of UTI isolates of $E$. faecalis revealed that $t c p F$ is more frequent among isolates of human urinary tract infections than stool flora, particularly in isolates with bacterial counts of more than $10 \mathrm{e} 5 \mathrm{cfu} / \mathrm{mL}(P=0.028)$. In vitro, $\mathrm{TcpF}$ was shown to impair the secretion of proinflammatory cytokines during infection of host cells and to interfere with TLR2 signaling upon intracellular expression, supporting the concept of molecular mimicry, as imposed to this group of proteins. Due to TcpF's ability to negatively modulate recognition by the TLR2 receptor and NF- $\kappa \mathrm{B}$ activation and the fact that $t c p F$ not only is common among $E$. faecalis isolates from intestinal flora but even prevails among human UTI isolates (Figure 1), it appears that $t c p F$ represents a recently acquired adaptive feature for E. faecalis that favors UT invasion and generally benefits mucosa colonization.

\section{Conflict of Interests}

The authors declare that there is no conflict of interests regarding the publication of this paper.

\section{Acknowledgments}

The study was funded by grants from the Bundesministerium für Bildung und Forschung (BMBF) through the National Genome Research Network (NGFN) to Trinad Chakraborty and the Government of the State of Hesse (LandesOffensive zur Entwicklung Wissenschaftlich-ökonomischer Exzellenz-LOEWE-Männliche Infertilität bei Infektion und Entzündung-MIBIE) to Svetlin Tchatalbachev.

\section{References}

[1] M. S. Jin and J. Lee, "Structures of the toll-like receptor family and its ligand complexes," Immunity, vol. 29, no. 2, pp. 182-191, 2008. 
[2] C. Brikos and L. A. J. O'Neill, "Signalling of toll-like receptors," Handbook of Experimental Pharmacology, no. 183, pp. 21-50, 2008.

[3] Y. Kim, M. M. Brinkmann, and H. L. Ploegh, "TLRs bent into shape," Nature Immunology, vol. 8, no. 7, pp. 675-677, 2007.

[4] H. Kumar, T. Kawai, and S. Akira, "Toll-like receptors and innate immunity," Biochemical and Biophysical Research Communications, vol. 388, no. 4, pp. 621-625, 2009.

[5] R. M. Newman, P. Salunkhe, A. Godzik, and J. C. Reed, "Identification and characterization of a novel bacterial virulence factor that shares homology with mammalian toll/interleukin-1 receptor family proteins," Infection and Immunity, vol. 74, no. 1, pp. 594-601, 2006.

[6] C. Cirl, A. Wieser, M. Yadav et al., "Subversion of Toll-like receptor signaling by a unique family of bacterial Toll/interleukin-1 receptor domain-containing proteins," Nature Medicine, vol. 14, no. 4, pp. 399-406, 2008.

[7] G. A. Snyder, C. Cirl, J. Jiang et al., "Molecular mechanisms for the subversion of MyD88 signaling by TcpC from virulent uropathogenic Escherichia coli," Proceedings of the National Academy of Sciences of the United States of America, vol. 110, no. 17, pp. 6985-6990, 2013.

[8] G. K. Radhakrishnan and G. A. Splitter, "Biochemical and functional analysis of TIR domain containing protein from Brucella melitensis," Biochemical and Biophysical Research Communications, vol. 397, no. 1, pp. 59-63, 2010.

[9] G. K. Radhakrishnan, Q. Yu, J. S. Harms, and G. A. Splitter, "Brucella TIR domain-containing protein mimics properties of the toll-like receptor adaptor protein TIRAP," Journal of Biological Chemistry, vol. 284, no. 15, pp. 9892-9898, 2009.

[10] S. P. Salcedo, M. I. Marchesini, H. Lelouard et al., "Brucella control of dendritic cell maturation is dependent on the TIRcontaining protein Btpl," PLoS Pathogens, vol. 4, no. 2, article e21, 2008.

[11] F. Askarian, N. M. van Sorge, M. Sangvik et al., "A staphylococcus aureus TIR domain protein virulence factor blocks TLR2mediated NF- $\kappa$ B signaling," Journal of Innate Immunity, vol. 6 , no. 4, pp. 485-498, 2014.

[12] S. L. Chan, L. Y. Low, S. Hsu et al., "Molecular mimicry in innate immunity: crystal structure of a bacterial TIR domain," The Journal of Biological Chemistry, vol. 284, no. 32, pp. 2138621392, 2009.

[13] E. Domann, T. Hain, R. Ghai et al., "Comparative genomic analysis for the presence of potential enterococcal virulence factors in the probiotic Enterococcus faecalis strain Symbioflor 1," International Journal of Medical Microbiology, vol. 297, no. 78, pp. 533-539, 2007.

[14] M. Fritzenwanker, C. Kuenne, A. Billion et al., "Complete genome sequence of the probiotic enterococcus faecalis symbioflor 1 clone DSM 16431," Genome Announcements, vol. 1, no. 1, 2013.

[15] F. Sievers, A. Wilm, D. Dineen et al., "Fast, scalable generation of high-quality protein multiple sequence alignments using Clustal Omega," Molecular Systems Biology, vol. 7, article 539, 2011.

[16] Y. Zhang and J. Skolnick, "TM-align: a protein structure alignment algorithm based on the TM-score," Nucleic Acids Research, vol. 33, no. 7, pp. 2302-2309, 2005.

[17] S. Schäferkordt and T. Chakraborty, "Vector plasmid for insertional mutagenesis and directional cloning in Listeria spp.," BioTechniques, vol. 19, no. 5, pp. 720-722, 724-725, 1995.
[18] S. Machata, S. Tchatalbachev, W. Mohamed, L. Jänsch, T. Hain, and T. Chakraborty, "Lipoproteins of Listeria monocytogenes are critical for virulence and TLR2-mediated immune activation," The Journal of Immunology, vol. 181, no. 3, pp. 2028-2035, 2008.

[19] L. A. J. O'Neill, “The interleukin-1 receptor/Toll-like receptor superfamily: 10 Years of progress," Immunological Reviews, vol. 226, no. 1, pp. 10-18, 2008.

[20] J. K. Gautam, L. D. Comeau, J. K. Krueger, and M. F. Smith Jr., "Structural and functional evidence for the role of the TLR2 DD loop in TLR1/TLR2 heterodimerization and signaling," Journal of Biological Chemistry, vol. 281, no. 40, pp. 30132-30142, 2006.

[21] Q. Zhang, C. M. Zmasek, X. Cai, and A. Godzik, "TIR domain-containing adaptor SARM is a late addition to the ongoing microbe-host dialog," Developmental and Comparative Immunology, vol. 35, no. 4, pp. 461-468, 2011.

[22] I. G. Sava, E. Heikens, and J. Huebner, "Pathogenesis and immunity in enterococcal infections," Clinical Microbiology and Infection, vol. 16, no. 6, pp. 533-540, 2010.

[23] D. Mathai, R. N. Jones, and M. A. Pfaller, "Epidemiology and frequency of resistance among pathogens causing urinary tract infections in 1,510 hospitalized patients: a report from the SENTRY Antimicrobial Surveillance Program (North America)," Diagnostic Microbiology and Infectious Disease, vol. 40, no. 3, pp. 129-136, 2001.

[24] D. Hartl and E. Jones, Genetics, Analysis of Genes and Genomes, Jones and Bartlett, Burlington, Mass, USA, 7th edition, 2009.

[25] C. A. Arias and B. E. Murray, "The rise of the Enterococcus: beyond vancomycin resistance," Nature Reviews Microbiology, vol. 10, no. 4, pp. 266-278, 2012.

[26] O. Shibolet and D. K. Podolsky, "TLRs in the Gut. IV. Negative regulation of Toll-like receptors and intestinal homeostasis: addition by subtraction," The American Journal of Physiology: Gastrointestinal and Liver Physiology, vol. 292, no. 6, pp. G1469G1473, 2007.

[27] D. Franchimont, S. Vermeire, H. El Housni et al., "Deficient host-bacteria interactions in inflammatory bowel disease? the toll-like receptor (TLR)-4 Asp299gly polymorphism is associated with Crohn's disease and ulcerative colitis," Gut, vol. 53, no. 7, pp. 987-992, 2004.

[28] H. Clevers, "At the crossroads of inflammation and cancer," Cell, vol. 118, no. 6, pp. 671-674, 2004. 

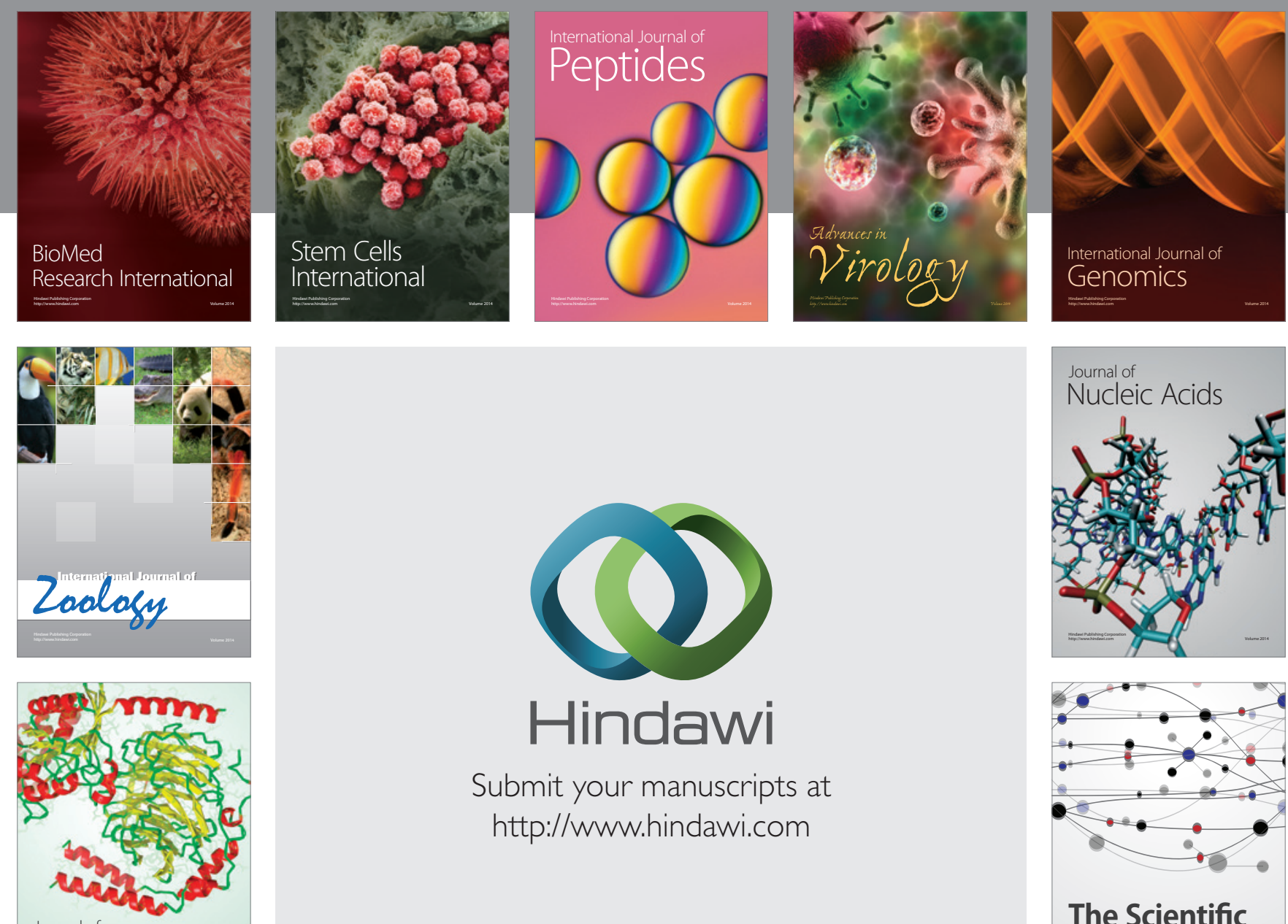

Submit your manuscripts at

http://www.hindawi.com

Journal of
Signal Transduction
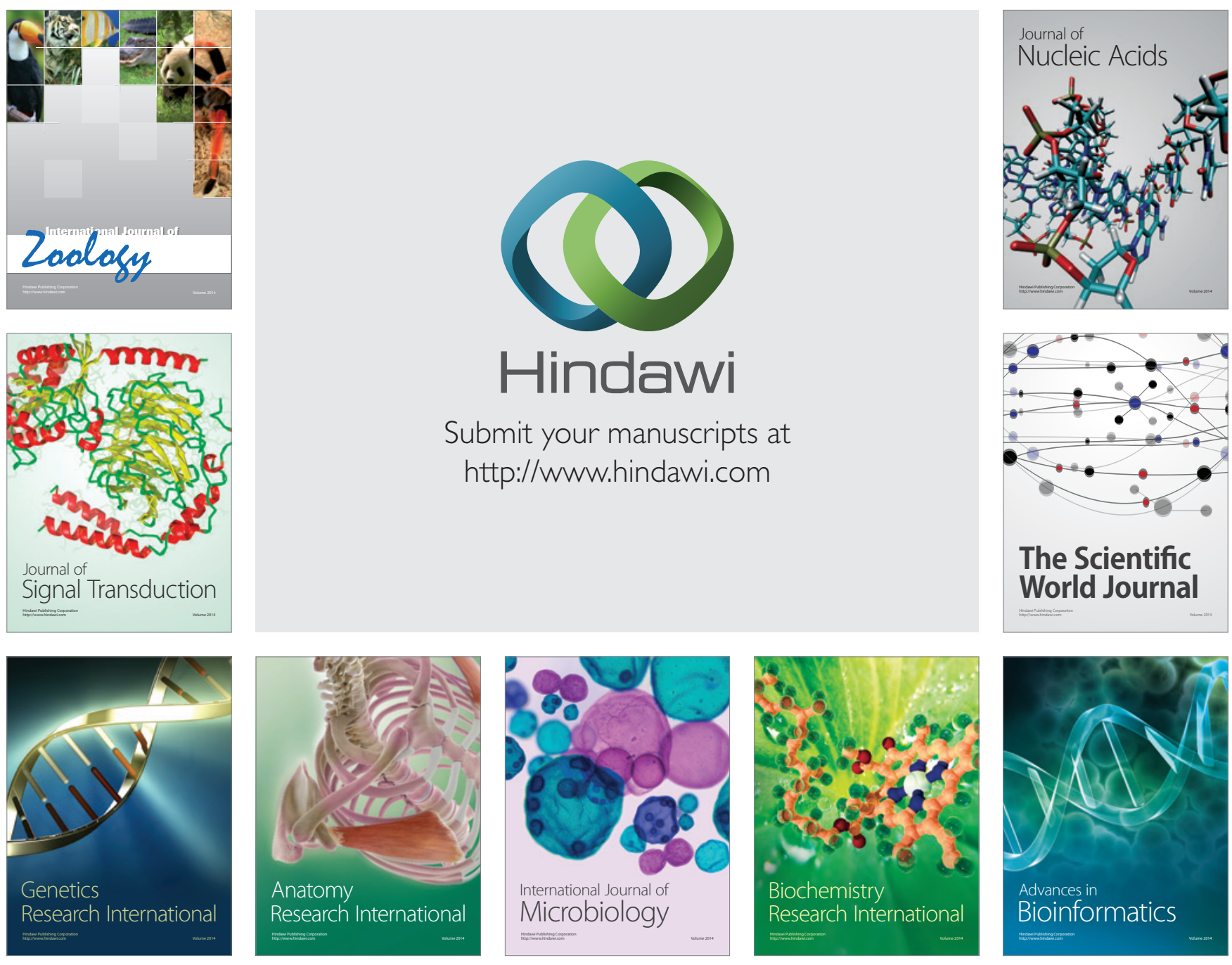

The Scientific World Journal
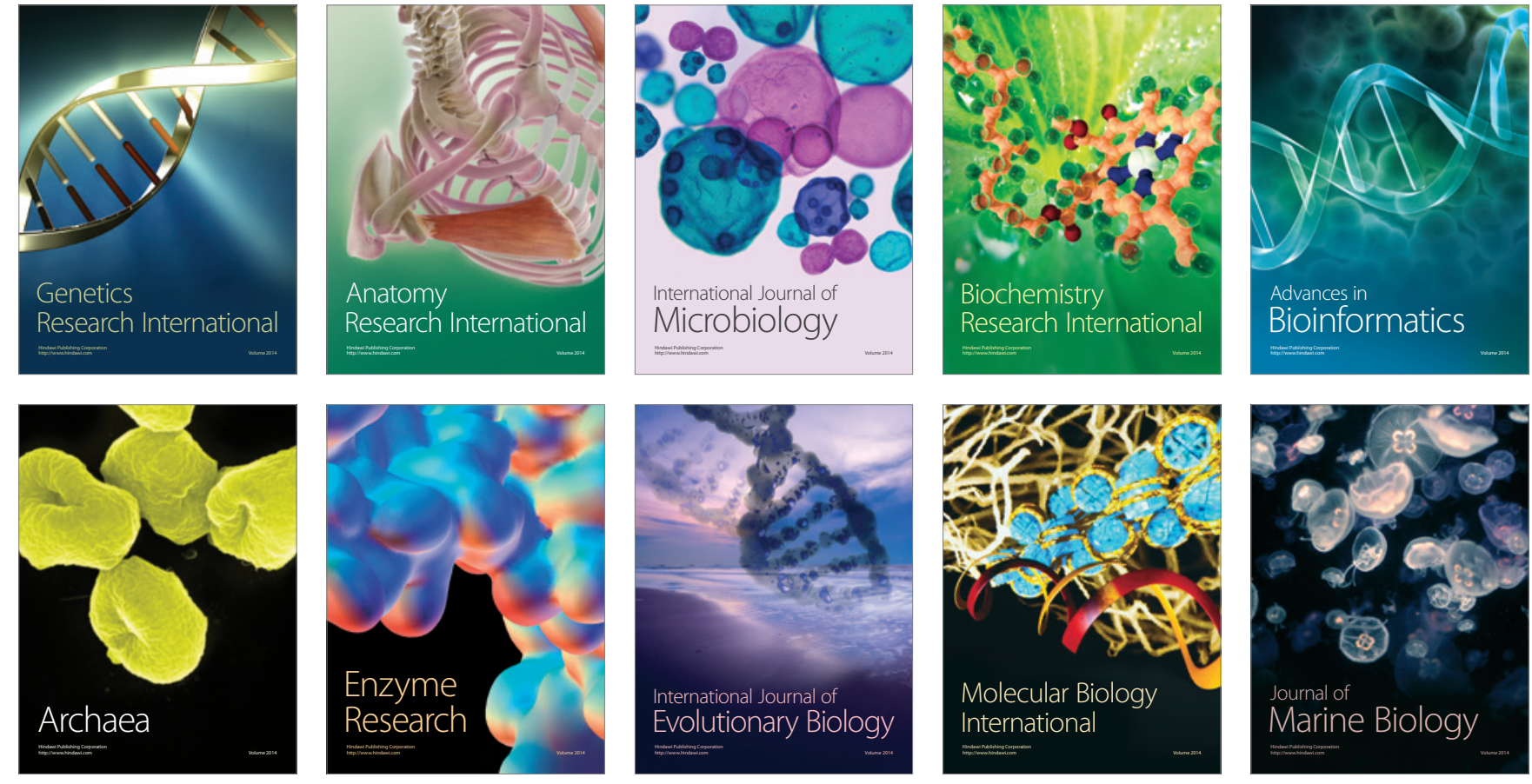